\title{
Desarrollo de un procedimiento constructivo más seguro para invernaderos multitúnel
}

\author{
A safer method of constructing multi-span greenhouses
}

$\underline{\text { A. Carreño }}^{(*)}$, J. Pérez ${ }^{(*)}$, J. Vázquez ${ }^{(*)}$, A. J. Callejón ${ }^{(*)}$

\section{RESUMEN}

Un nuevo sistema constructivo para invernaderos multitúnel ha sido desarrollado y evaluado. El nuevo sistema se fundamenta en el montaje del conjunto de la estructura de cubierta a nivel de suelo y posteriormente su elevación, hasta alcanzar su posición definitiva.

Para su evaluación, se ha construido un invernadero combinando ambos sistemas constructivos. La mitad del invernadero se ha ejecutado según el sistema tradicional y la restante según el nuevo procedimiento, empleando en ambos casos los mismos operarios.

Los parámetros registrados, horas de trabajo en altura, mano de obra empleada, necesidades de maquinaria complementaria y tiempo de montaje, ponen de manifiesto las importantes mejoras en cuanto a seguridad que conlleva la aplicación del nuevo procedimiento constructivo, que permite concluir que la siniestralidad por caídas en altura se reduciría un 9,2\% debido a la disminución de las horas de trabajo en altura en un $40 \%$.

845-16

Palabras clave: Construcción, diseño, cubierta, invernadero, seguridad.

\section{SUMMARY}

This paper reports the development and testing of a new procedure for constructing multi-span greenhouses, based on the assembly of the roof structure at ground level and later raising it to its final position.

To compare the proposed and traditional construction procedures, a greenhouse was assembled employing each method to erect one half of the final structure. The same workers erected both halves.

The proposed method showed important advantages in terms of the time spent working at height, the number of workers required, the complementary machinery needed, and the overall assembly time, thus reducing the risk of accidents, concluding that fall from height will be reduced $9,2 \%$ due to a reduction of working hours at height in $40 \%$

Keywords: Construction, design, roof, greenhouse, safety. 


\section{INTRODUCCIÓN}

España es el país más importante en el uso de invernaderos de cubierta plástica (1), Ilegando hasta las 45.000 ha (2) de las que la mayor parte se concentran en el sureste peninsular, destacando Almería como la provincia con mayor superficie invernada con 25.983 ha (3). Estos invernaderos se destinan al cultivo de hortalizas, fundamentalmente durante las estaciones de otoño, invierno y primavera (4).

Los invernaderos se constituyen con estructuras ligeras, de bajo coste, adaptadas a los requerimientos climáticos (5), siendo el modelo de invernadero tradicionalmente empleado en el sureste español el denominado "tipo parral", aunque en estas últimas décadas está siendo sustituido por otros más perfeccionados, como el Ilamado "raspa y amagado" y el multitúnel, que posibilitan un mejor control climático así como posibilidades de automatización (6).

La época estival, dada la ausencia de cultivo, suele ser la habitual para desarrollar los trabajos de construcción y mantenimiento de las estructuras invernadas en la provincia almeriense, produciéndose una fuerte demanda de mano de obra que se solventa con la contratación, en ocasiones, de operarios con carencias en formación y experiencia. Respecto a este hecho, diversos estudios han puesto de manifiesto que el empleo de mano de obra no especializada puede ser la causa desencadenante de accidentes laborales (7-9).

En Almería existen 23 empresas dedicadas a la construcción de invernaderos con una media de 13,46 trabajadores por empresa (10), pudiendo clasificarlas como pequeñas empresas en función de su número de trabajadores. Varios estudios afirman que las empresas de pequeño tamaño tienen mayor probabilidad de sufrir accidentes, dado que poseen mayores limitaciones en cuanto a recursos preventivos (11-15).

En la actualidad son muchas las investigaciones sobre seguridad laboral en construcción, abordando preferentemente las edificaciones y construcciones industriales (16-21), siendo escasos los estudios específicos sobre construcción de invernaderos (22-23), limitándose las publicaciones existentes a citar metodologías y describir tipologías constructivas (24-28), aunque sí se hace perceptible, la presencia de estudios sobre ergonomía en entornos laborales de invernaderos (29).

La siniestralidad en la construcción de invernaderos se encuadra dentro del sec- tor de la construcción a efectos estadísticos para las diferentes administraciones responsables de la prevención, estudio y registro de los accidentes laborales y enfermedades profesionales. El Índice de Incidencia en Construcción de Invernaderos, promedio del periodo 2000-2006, fue de 154,02, siendo superior al del sector de la construcción en Almería, que fue de 144,45 y de Andalucía que fue de 151,66. Las principales causas de siniestralidad son sobreesfuerzos, contacto con agentes materiales cortantes, caídas de personal a distinto nivel, aplastamientos y golpes por objetos o herramientas, siendo las regiones anatómicas más lesionadas la espalda y miembros inferiores. Por importancia, las caídas en altura resultan ser los riesgos más dañinos con una incidencia del $23 \%$ (30).

Los sistemas empleados en la construcción de invernaderos son bastante rudimentarios, siendo lo más habitual el empleo por parte de los trabajadores de los propios elementos estructurales como base de apoyo para la ejecución de las restantes labores. Este sistema carece de medidas de protección alguna para controlar los riesgos a los que se expone el trabajador.

Otro de los sistemas empleados, se basa en el uso de carretillas elevadoras en las que el obrero trabaja sobre un palet. Este procedimiento adolece de evidentes carencias en materia de seguridad, donde el operario apenas tiene capacidad de movimiento sobre la plataforma, sumando a ello la ausencia de rodapiés, barandillas de sujeción u otro medio de protección.

Por otra parte, algunos constructores, acoplan estrechas plataformas sobre los laterales del cajón de carga de vehículos tipo camión, siendo este sistema más estable que el anterior, pero con ausencia de medidas de protección.

Un sistema mejorado frente a todos los anteriores, consiste en el empleo de plataformas elevadoras que incorporan vallas de protección y rodapiés, pero sin embargo, este sistema no se ha implantado completamente debido al elevado coste que implica su arrendamiento, la ralentización del trabajo efectuado en altura, además de problemas adicionales de desplazamiento sobre suelos enarenados. Asimismo, en ocasiones, su empleo no garantiza la prevención de accidentes, ya que su utilización no siempre es la adecuada (31).

En general, podemos decir que los métodos actuales de construcción de invernaderos de estructura metálica ligera conllevan una serie de riesgos derivados fundamental- 
mente del trabajo en altura de los operarios, comprobándose que en la mayoría de los casos, las medidas propuestas normativamente para evitar o reducir dichos riesgos no son tenidas en cuenta, justificándose aún más la necesidad de cambiar los sistemas constructivos, tendiendo hacia procedimientos de diseño más preventivos.

En este sentido, son muchos los autores que consideran el diseño como una herramienta clave para evitar o, en su caso, reducir riesgos (32-38). Otros autores (39), promueven el desarrollo de nuevas técnicas de gestión de la calidad en la industria de la construcción. En estas líneas de trabajo, el objetivo de nuestra investigación es desarrollar y evaluar una nueva metodología constructiva para invernaderos multitúnel orientada a minimizar las horas de trabajo en altura y de esa forma disminuir los riesgos laborales asociados.

\section{MATERIALES Y MÉTODOS}

\subsection{Sistema constructivo actual}

El proceso constructivo tradicional comprende la realización de las siguientes etapas $(31 ; 40)$ :

1. Desbroce y nivelación del terreno. El suelo debe estar limpio y, preferiblemente, nivelado para comenzar a desarrollar los trabajos.

2. Replanteo del invernadero (tira de cuerdas). Durante esta etapa se efectúa la señalización de los pilares sobre el terreno.

3. Apertura de los hoyos de cimentación. La cimentación de estos invernaderos se realiza mediante pilotes cilíndricos de $1 \mathrm{~m}$ de profundidad y $0,3 \mathrm{~m}$ de diámetro. La ejecución de las excavaciones se realiza por medio de una perforadora/ ahoyadora de accionamiento hidráulico o mediante rotación.

4. Disposición de hormigón de limpieza y, una vez fraguado éste, replanteo nuevamente del conjunto de los pilares, tanto en planimetría como altimetría.

5. Disposición de pilares garantizando su correcta verticalidad y alineación, seguido del hormigonado de pilotes.

6. Montaje de estructura de cubierta. El proceso comprende la realización de las siguientes tareas:

a) Disposición de capiteles en cabeza de pilares. Una vez fraguado el hormigón vertido en los pilotes y por tanto con los pilares fijados correctamente al suelo, se procede con la disposición de los capiteles. Se trata de elementos metálicos que actúan como nudos enlazando el conjunto de arcos y barras de cubierta que confluyen en ese punto (cabeza pilar), transmitiendo el conjunto de esfuerzos a los pilares. b) Montaje de canalones sobre capiteles. Los canalones son elementos estructurales con disposición perpendicular a los pórticos, que enlazan las cabezas de los pilares y que posibilitan la evacuación de aguas pluviales. Para evitar el goteo de agua en el interior del invernadero, es necesario realizar correctamente el solape entre canalones con la disposición de una junta elástica.

c) Montaje de arcos. Para tal fin se emplean los capiteles como elementos de apoyo. Los arcos son perfiles circulares huecos curvados que determinan la forma de la cubierta, ya que según el tipo de arco que utilicemos la tipología de invernadero será distinta, así encontramos los invernaderos multitúnel, góticos, asimétricos, etc. La unión de los arcos al nudo-capitel se realiza por acople de los extremos del arco a los capiteles mediante abrazaderas atornilladas.

d) Montaje de pendolones y barras de cultivo en pórticos principales. Los pendolones son los elementos que arriostran la estructura en el plano de la misma, equivalente a los montantes de una cercha. La unión se realiza mediante abrazaderas tanto en su parte superior (unión con el arco), como en su parte inferior (unión a la barra de cultivo). Por otra parte, la barra de cultivo es un elemento estructural equivalente al tirante de una cercha, unido en sus extremos a los capiteles mediante tornillería. Su nombre se debe a que dichos elementos son empleados para soportar el peso propio de los cultivos de enrame.

e) Montaje de perfiles longitudinalmente a modo de correas en cubierta. Se trata de perfiles conformados en forma de $\mathrm{C}$ o $\mathrm{H}$ con dimensiones aproximadas de $3 \mathrm{~cm}$ de canto que enlazan, con tornillería, a los arcos. Estos elementos se han diseñado con esta forma para posibilitar la fijación del film plástico de cubierta.

f) Montaje de elementos complementarios (pies inclinados, cruces de San Andrés, etc.) para soporte de la acción de viento en paramentos perimetrales. Los invernaderos suelen estar reforzados perimetralmente frente a la acción del viento mediante la utilización de barras y/o tensores que reducen las deformaciones. Algunos de estos elementos son específicos para la cubierta, rigidizando los pórticos extremos.

g) Disposición del film plástico de cubierta. Esta labor se realiza de forma totalmente manual empleando las correas como elementos de fijación. La sujeción del film plástico se realiza mediante unas piezas cilíndricas y huecas de polietileno, denominadas coloquialmente tacos, que encajan mediante golpeo en el interior de las correas, siendo fijadas a las mismas mediante tornillería. 
1. Sistema constructivo tradicional con auxilio de plataforma

2. Construcción de túnel con el nuevo procedimiento constructivo.
Resulta destacable (Figura 1) que el ensamblado de la estructura de cubierta es la labor más dificultosa, en tiempo y recursos, ya que se desarrolla en su totalidad a alturas comprendidas entre los 4-8 m.

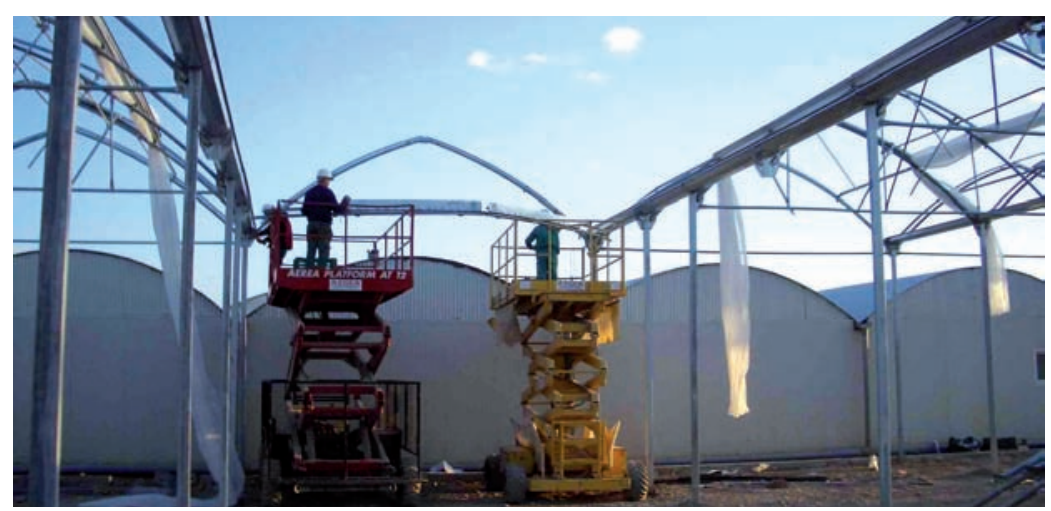

2.2. Antecedentes del prototipo ensayado

Para alcanzar el objetivo propuesto, se planteó realizar el montaje de la estructura de cubierta del invernadero a nivel de suelo (Figura 2), y no a más de $4 \mathrm{~m}$ como en el sistema tradicional, para posteriormente elevarla ayudándose de sistemas motrices.

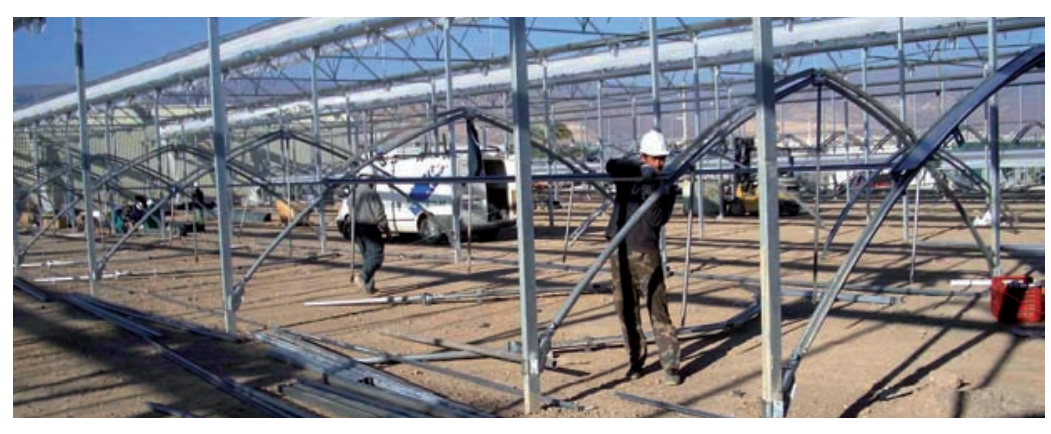

Ante este planteamiento, surgió la primera alternativa de trabajo importante:

a) subir el conjunto de la estructura de cubierta del invernadero de forma solapada.

b) subir la estructura de cubierta de cada túnel independientemente.

En cuanto al sistema de elevación, también se planteó el estudio de diversas variantes:

a) colocar los motores de elevación en cabeza de pilar.

b) colocar los motores de elevación en la base de los pilares.

El conjunto de las alternativas fueron estudiadas con la realización de pruebas a tres escalas:

1. Pruebas a escala de taller/laboratorio. Boceto.

2. Pruebas a escala reducida o de maqueta.

3. Pruebas a dimensión real del prototipo.
Este procedimiento de ensayo a tres escalas, permitió tomar decisiones técnicas en cada etapa, tanto del funcionamiento como de las mejoras a realizar en las piezas, de forma que en la tercera etapa (dimensión real) se ensayó un modelo prácticamente definitivo basado en la ascensión de los diferentes túneles del invernadero de forma independiente, con la disposición de los motores de elevación en la base de los pilares.

Los criterios considerados para la selección de alternativas han sido: riesgo y coste. El riesgo ha sido evaluado con la cuantificación de las horas de trabajo en altura y el coste con la necesidad de plataformas elevadoras y con el número de equipos motrices necesarios.

La decisión del izado independiente de los túneles se fundamentó en reducir el peso total a elevar, facilitando el proceso de izado y las posteriores tareas de fijación de la estructura de cubierta a los pilares, optimizando el uso de los recursos empleados por el sistema de motriz. Todos los elementos constituyentes del sistema motriz se diseñaron con la premisa de poder ser retirados con facilidad para su posterior reutilización en los siguientes túneles del invernadero. Por tanto, para la toma de esta decisión, el criterio de selección más importante fue el coste, siendo el criterio de riesgo similar en ambas alternativas. En el caso de subir todos los túneles del invernadero de forma simultánea, se precisaría de un mayor número de equipos motrices (motores, ejes, carretes de enrollamiento, poleas en T, etc.), lo cual implicaría un incremento de coste.

Por otra parte, dado que la colocación del sistema motriz (eje, carretes y motores) en el extremo superior del pilar, implicaba, incluso en la etapa de pruebas a escala reducida, considerables horas de trabajo en altura, se decidió disponerlo en la base de los pilares. Así se logró reducir el número de horas de trabajo en altura, a costa de diseñar un nuevo elemento: el sistema de poleas en T. Los criterios de selección considerados en esta alternativa fueron tanto el riesgo como el coste. Evidentemente, seleccionando la alternativa de colocación del sistema motriz en la parte inferior de los pilares se reduce el riesgo por trabajo en altura, disminuyendo también la necesidad de plataformas elevadoras, y, con ello, el coste asociado.

De esta forma, se consigue que el montaje del conjunto de los elementos de cubierta se efectúe a nivel del suelo, quedando reducidas las operaciones a realizar en altura a la disposición de canalones, el montaje de elementos puntuales del sistema de elevación (sistema de poleas en T), la fijación de 
los elementos de cubierta a los pilares (fijación de capiteles) y finalmente, en parte, la disposición del film plástico de cubierta. El nuevo procedimiento modifica las labores de montaje de los elementos de cubierta (Tabla 1), resultando las labores previas de acondicionamiento del terreno y cimentación de pilares semejantes a las tradicionalmente efectuadas.
Otro de los elementos que resulta destacable por su novedad, son los capiteles, ya que tradicionalmente se vienen empleando capiteles conformados por una única pieza $(41,42)$, mientras que con el nuevo procedimiento, los túneles se izan independientemente y se ensamblan una vez alcanzada su posición definitiva, precisando para ello de capiteles partidos, también denominados semi-capiteles (43).
3. Esquema del nuevo procedimiento constructivo.

Tabla 1

Comparativa de sistemas de montaje

\begin{tabular}{|l|c|c|}
\hline \multicolumn{2}{|c|}{ MONTAJE DE ESTRUCTURA DE CUBIERTA } \\
\hline & $\begin{array}{c}\text { Procedimiento } \\
\text { tradicional }\end{array}$ & $\begin{array}{c}\text { Nuevo } \\
\text { procedimiento }\end{array}$ \\
\hline Montaje de canalones en altura & $X$ & $X$ \\
\hline Montaje de elementos de cubierta en suelo & & $X$ \\
\hline Montaje de mecanismo de elevación a nivel de suelo & & $X$ \\
\hline Elevar elementos de cubierta & & $X$ \\
\hline Enlazar la cubierta a los pilares en altura & $X$ & $X$ \\
\hline $\begin{array}{l}\text { Disposición de elementos de cubierta en altura con } \\
\text { auxilio de plataformas elevadoras }\end{array}$ & $X$ & \\
\hline Colocación de elementos complementarios en altura & $X$ & \\
\hline Disposición del film plástico & $X$ & $X$ \\
\hline
\end{tabular}

\subsection{Componentes del nuevo procedimiento}

Para elevar la estructura de cubierta se precisa de un sistema motriz que imprima fuerza suficiente para ascenderla (Figura 3). El sistema consta de un motor con caja reductora de potencia $900 \mathrm{~W}$ capaz de hacer girar un eje motriz que se dispone a lo largo de todo el túnel próximo al suelo, con una velocidad angular de 2,6 rpm. Este eje se une a la base de los pilares mediante el uso de palieres con rodamientos que permiten el giro con apenas rozamiento. Al eje motriz se le une de forma solidaria un carrete de enrollamiento que permitirá recoger el cable de tracción al ir girando el tubo motriz y ascendiendo la estructura, de forma que el cable de tracción comienza en el carrete de enrollamiento y finaliza en la barra de cultivo a la que se une mediante una abrazadera y gancho en S. Intercalado en este sistema se encuentra un tensor que permite ajustar la tensión adecuada para poder ascender la estructura de forma equilibrada en todos los arcos.

Para que el cable de tracción pueda pasar por encima del canalón transmitiendo toda la fuerza que le imprime el sistema motoreductor, hace falta la colocación de un sistema de poleas en la parte superior del canalón. Este sistema se ha diseñado en forma de $T$ para que no se pierda la perpendicularidad del cable de tracción, intercalando en su estructura dos poleas que modifican la dirección del cable de tracción (Figura 3).

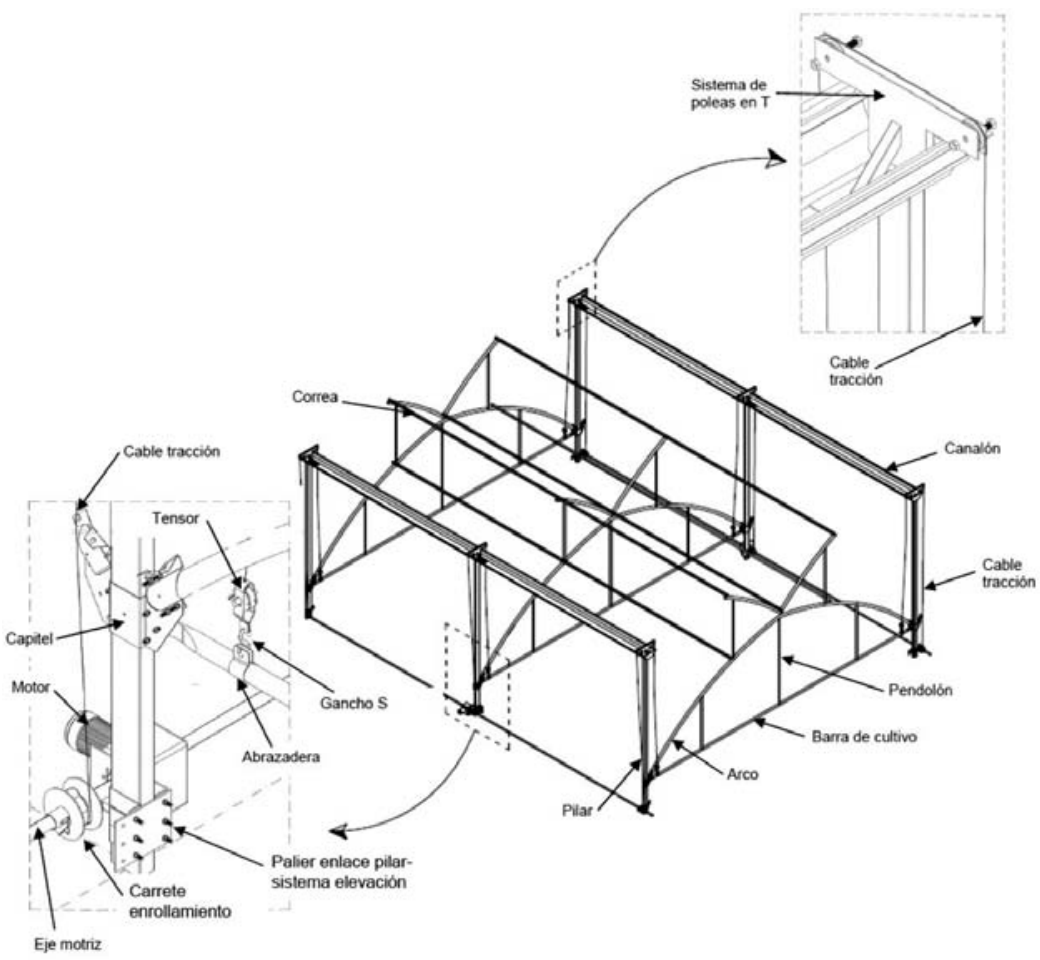

\subsection{Ensayos experimentales}

Para el desarrollo de los ensayos del prototipo definitivo, se ha construido a escala real un invernadero en el campo experimental de la Escuela Técnica de Investigación y Formación Agraria (ETIFA), ubicada en la provincia de Almería (Figura 4). Se trata de un invernadero multitúnel en su variante "tipo gótico" con doble ventana cenital. El modelo presen- 
4. Estructura de cubierta de túnel de invernadero experimental montado a la espera de su elevación. ta luces de $8 \mathrm{~m}$ y separación entre pórticos de $5 \mathrm{~m}$, con arcos flotantes intercalados cada $5 \mathrm{~m}$. La altura hasta canalón es de 4,5 m resultando ser la altura cenital de $7 \mathrm{~m}$.

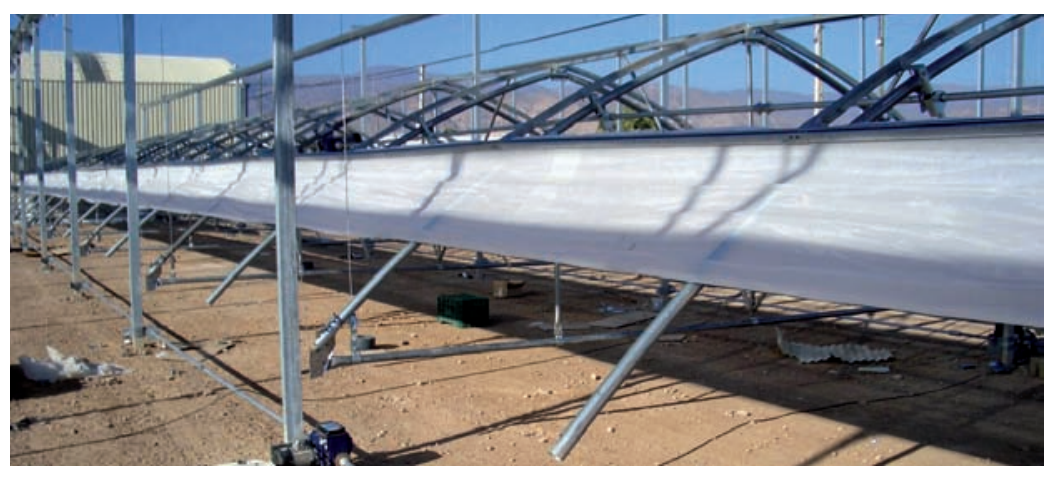

El invernadero se compone de 10 túneles iguales de $78 \mathrm{~m}$ de longitud, con una superficie total en planta de $6.240 \mathrm{~m}^{2}$.

La mitad del invernadero se ha construido según el sistema tradicional y la restante según el nuevo procedimiento, usando en ambos casos los mismos operarios, montadores adiestrados. Así se logra reducir la posible variabilidad producida por la utilización de distintas cuadrillas de trabajadores en un sistema constructivo y en otro.

Concretamente, el primero, tercero, quinto, séptimo y noveno túnel se construyó según el nuevo procedimiento, mientras que en el segundo, cuarto, sexto, octavo y décimo túnel, utilizando el método tradicional.

De esta forma, se pueden efectuar comparaciones en el consumo de mano de obra y maquinaria entre ambos sistemas, eliminando posibles distorsiones producidas por otros factores, como pueden ser diferencias de cualificación, experiencia y rendimiento en el trabajo entre operarios, etc.

La evaluación de ambos sistemas, se efectuó mediante el registro diario de las operaciones realizadas, así como el tiempo y los recursos empleados en cada una de ellas.

Las variables analizadas, expresadas por hectárea invernada, han sido las siguientes:

- Horas de trabajo en altura.

- Mano de obra empleada.

- Uso de maquinaría.

- Tiempo de montaje.

\section{RESULTADOS}

Se han registrado las horas de trabajo en altura del sistema tradicional frente al propuesto (Figura 5), sólo en aquellas operaciones en las que existen diferencias entre ambos sistemas. Es destacable que el procedimiento propues- to evita la labor de montaje de los elementos de cubierta en altura, reduciendo los tiempos de montaje de las labores de colocación de canalones y plástico. En cambio, el sistema propuesto requiere la realización de dos nuevas labores, montar el mecanismo de elevación y abrochar el invernadero en altura. En definitiva, el nuevo procedimiento implica una reducción de horas de trabajo en altura de un $40 \%, 626,28$ horas $\cdot$ ha $^{-1}$ frente a las $1.044,20$ horas $\cdot$ ha $^{-1}$ del sistema tradicional.

Las necesidades de mano de obra (Figura 6) han sido registradas diferenciándolas por operaciones a realizar y nivel profesional, oficial o peón. Se hace perceptible que las necesidades son semejantes en las fases previas de replanteo, excavación, colocación de pilares y cimentación de pilotes, mientras que nuevamente, el montaje tradicional de los elementos de cubierta es la etapa de mayor demanda de personal, destacando la necesidad de oficiales, con 486,38 horas $\cdot \mathrm{ha}^{-1}$.

Por otra parte, las horas de empleo de mano de obra por hectárea, ponen de manifiesto importantes reducciones en el cómputo total de horas de trabajo, cifradas en un $14,74 \%$ en el caso de oficiales y un $7,65 \%$ en el caso de peones.

En cuanto a las necesidades de maquinaría, es destacable tan sólo el empleo de plataformas elevadoras. Se han registrado las horas de trabajo con plataformas elevadoras en ambos sistemas, desagregándolas por operaciones constructivas (Figura 7), poniéndose de manifiesto los mayores requerimientos del sistema tradicional para efectuar el montaje de los elementos de cubierta $\left(482,12\right.$ horas $\cdot$ ha $\left.^{-1}\right)$, mientas que el nuevo procedimiento emplea este tipo de maquinaría principalmente para efectuar la fijación de los elementos de cubierta a los pilares $\left(225,71\right.$ horas $\cdot$ ha $\left.^{-1}\right)$. En el resto de las labores, comunes para ambos sistemas, los requerimientos del nuevo procedimiento son inferiores. De esta forma, el nuevo sistema logra reducir en un $44,89 \%$ las necesidades de plataformas elevadoras.

Por otra parte, la Figura 8 recoge los tiempos medios de montaje para ambos procedimientos desagregados por operaciones, resultando destacable que el nuevo procedimiento constructivo logra reducir en un 9,93\% el tiempo medio de montaje por hectárea.

A su vez, la Tabla 2 muestra el nivel de riesgo en la construcción de invernaderos multitúnel con el procedimiento tradicional. La evaluación, desarrollada con el procedimiento propuesto por el INSHT (44), identifica las caídas de personas a distinto nivel, la presencia de energías peligrosas y las condiciones ambientales inadecuadas, 

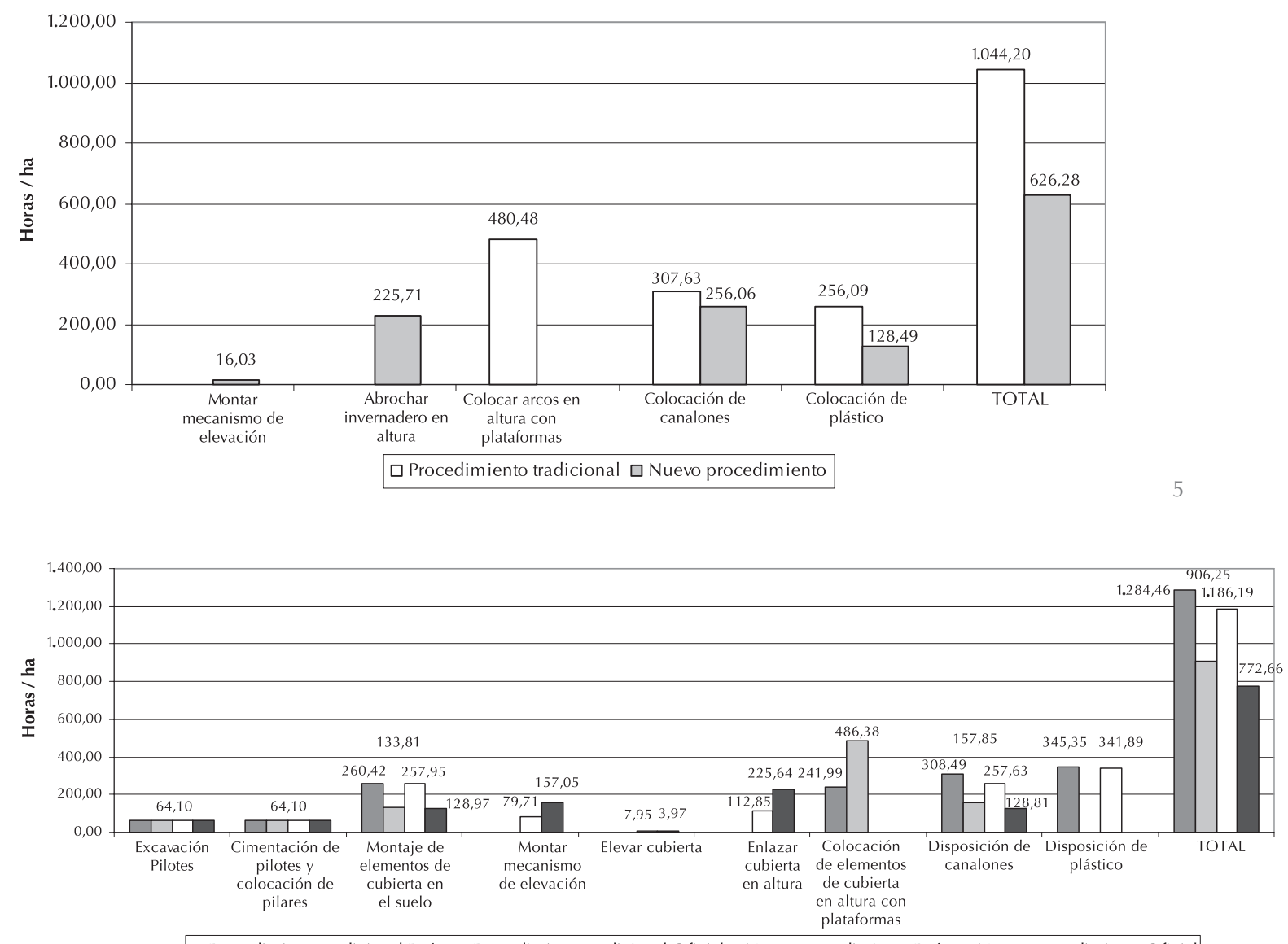

$\square$ Procedimiento tradicional Peón $\square$ Procedimiento tradicional Oficial $\square$ Nuevo procedimiento Peón $\square$ Nuevo procedimiento Oficial
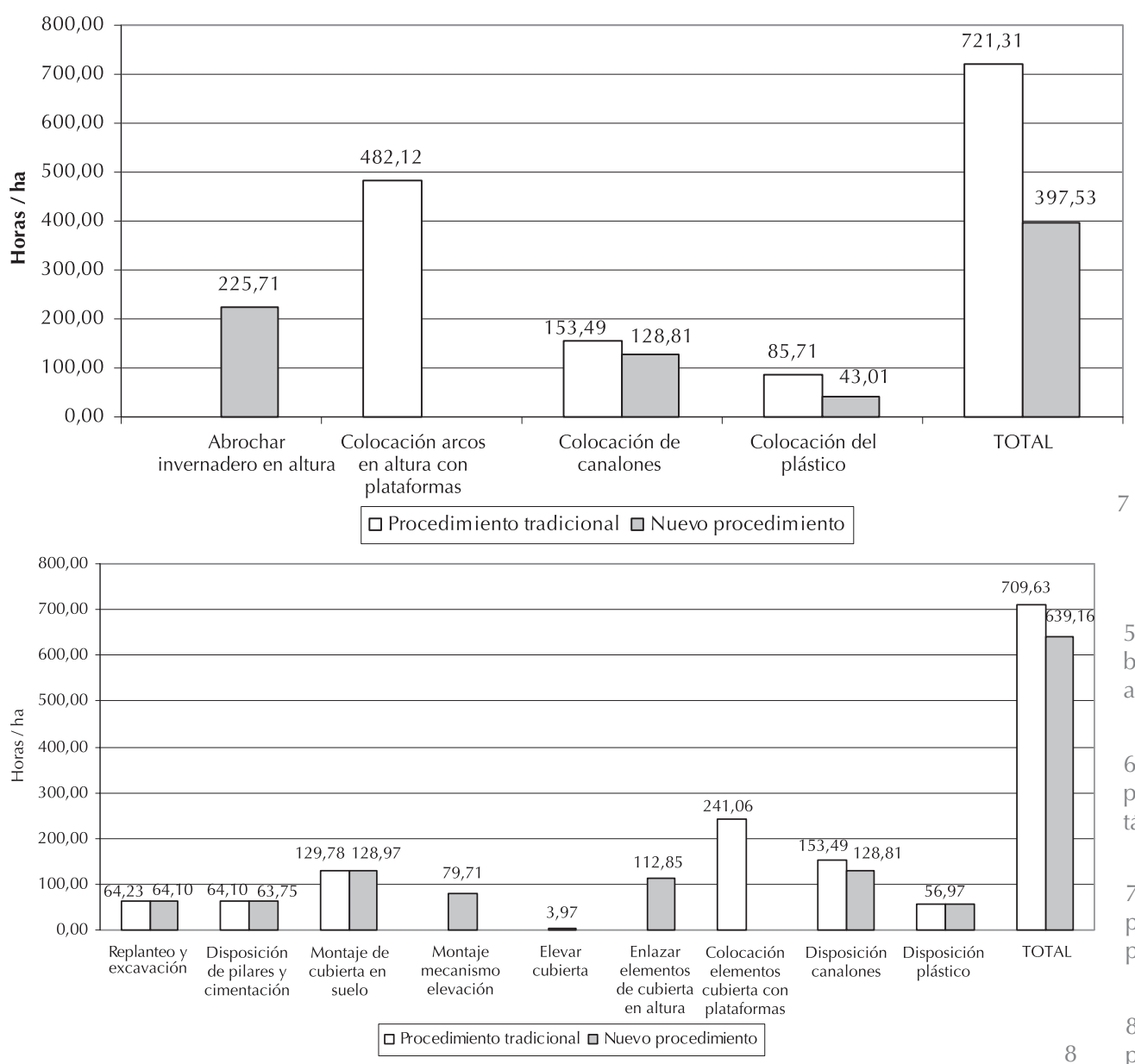

5. Promedio de horas de trabajo en altura por hectárea de ambos sistemas.

6. Promedio de horas de empleo de mano de obra por hectárea.

7. Promedio de horas de empleo de plataforma elevadora por hectárea.

8. Tiempo medio de montaje 8 por operaciones y hectárea. 
como los peligros con mayor nivel de riesgo. Las energías peligrosas, hacen referencia a la presencia de postes y tendidos eléctricos en las proximidades de los invernaderos.

Con el nuevo procedimiento, los peligros potenciales son los mismos que con el sistema tradicional, pero la diferencia reside en que el grado de exposición al peligro es mucho menor, y, por lo tanto, la probabilidad de que suceda se reduce. El montaje del conjunto de los elementos de cubierta (capiteles, arcos, pendolones, barras de cultivo, correas y elementos complementarios) y, parcialmente, la disposición del film plástico, se realiza a nivel del suelo, evitando así el peligro de caída de personas y objetos en altura. El montaje de los canalones, la disposición del sistema de poleas en T, la fijación de capiteles y parcialmente la disposición del film plástico de cubierta, se siguen realizando en altura, siendo los peligros y el nivel de riesgo, semejantes a los identificados con el sistema tradicional. Se considera que la colocación del sistema de poleas en T tiene un nivel de riesgo semejante a la disposición de canalones.
La aplicación del nuevo procedimiento constructivo reduce un $40 \%$ las horas de trabajo en altura, y dado que según Miranda-García y Martínez-López (2007) (30), el porcentaje de siniestros debidos a caídas en altura de los invernaderos es del $23 \%$, es previsible que el nuevo procedimiento conlleve una reducción de la siniestralidad debido a caídas en altura proporcional a la reducción de horas de trabajo en altura. Esta reducción se cifraría en una disminución del $40 \%$ del $23 \%$ del porcentaje que implican este tipo de siniestros. De esta forma podemos deducir que la aplicación del nuevo procedimiento constructivo reducirá en un 9,2\% (0,4 × 23\%) la siniestralidad laboral debida a caídas en altura.

Por otra parte, la reducción de mano de obra del nuevo procedimiento, $14,74 \%$ en el caso de oficiales y un $7,65 \%$ en el caso de peones, logra reducir la demanda de personal. Este aspecto resulta clave en el período punta de construcción y rehabilitación de invernaderos, la época estival, ya que según Camacho-Ferre (1999) (4) es la única estación donde no se cultivan hortalizas. Es en

Tabla 2

Nivel de riesgo en la construcción de invernaderos multitúnel con el procedimiento tradicial

\begin{tabular}{|c|c|c|c|c|c|}
\hline \multicolumn{6}{|c|}{$\begin{array}{l}\text { NIVEL DE RIESGO EN LA CONSTRUCCIÓN DE INVERNADEROS MULTITUNEL } \\
\text { "Procedimiento tradicional" }\end{array}$} \\
\hline \multirow[b]{2}{*}{$\begin{array}{l}\text { IDENTIFICACIÓN } \\
\text { DE PELIGROS }\end{array}$} & \multicolumn{5}{|c|}{ ETAPAS DEL PROCEDIMIENTO CONSTRUCTIVO } \\
\hline & $\begin{array}{c}\text { Colocación de } \\
\text { capiteles en altura } \\
\text { definitiva }\end{array}$ & $\begin{array}{l}\text { Colocación de } \\
\text { canalones }\end{array}$ & $\begin{array}{l}\text { Colocación de } \\
\text { arcos, pendolones } \\
\text { y barras de cultivo }\end{array}$ & $\begin{array}{l}\text { Colocación de } \\
\text { correas y elementos } \\
\text { complementarios }\end{array}$ & $\begin{array}{l}\text { Colocación } \\
\text { del plástico }\end{array}$ \\
\hline Golpes y cortes & Tolerable & Tolerable & Tolerable & Tolerable & Tolerable \\
\hline Caídas de personas a distinto nivel & Moderado & Moderado & Importante & Importante & Importante \\
\hline $\begin{array}{l}\text { Caída de herramientas, materiales, } \\
\text { etc., desde altura }\end{array}$ & Moderado & Moderado & Moderado & Moderado & Moderado \\
\hline $\begin{array}{l}\text { Peligros asociados con manejo } \\
\text { manual de cargas }\end{array}$ & Tolerable & Tolerable & Tolerable & Tolerable & Moderado \\
\hline $\begin{array}{l}\text { Peligros en las instalaciones y en } \\
\text { el manejo de máquinas durante el } \\
\text { montaje, consignación, operación, etc. }\end{array}$ & Moderado & Moderado & Moderado & Moderado & Moderado \\
\hline $\begin{array}{l}\text { Energías peligrosas (electricidad, } \\
\text { radiaciones y vibraciones) }\end{array}$ & $\begin{array}{l}\text { Importante } \\
\text { Moderado }\end{array}$ & $\begin{array}{l}\text { Importante } \\
\text { Moderado }\end{array}$ & $\begin{array}{l}\text { Importante } \\
\text { Moderado }\end{array}$ & $\begin{array}{l}\text { Importante } \\
\text { Moderado }\end{array}$ & $\begin{array}{l}\text { Importante } \\
\text { Moderado }\end{array}$ \\
\hline Ambiente térmico inadecuado & Importante & Importante & Importante & Importante & Importante \\
\hline
\end{tabular}

\section{DISCUSIÓN}

Completando los anteriores estudios sobre construcción de invernaderos realizados por Hoxey et al. (1993) (24), Matallana et al. (1995) (25), Tsirogiannis (1996) (26), Waaijenberg (1997) (27) y Mistriotis et al. (2002) (28), el nuevo procedimiento constructivo consigue, con las correspondientes modificaciones de diseño, importantes reducciones de siniestralidad. Estos resultados se asemejan a los obtenidos, en otros ámbitos, por Christensen (2003) (32), Behm (2005) (33), Lam et al. (2006) (34), Mrosczyzk (2006) (35), Van Gorp (2007) (36) y Gambatese et al. (2008) (37). esta época donde la demanda de mano de obra resulta ser en ocasiones excedentaria a la oferta del mercado. El mercado solventa estas deficiencias con la contratación de personal con escasa formación y experiencia, siendo entonces cuando mayor probabilidad de siniestros existe, coincidiendo con los resultados de Saha et al. (2004) (7), Waehrer et al. (2008) (8) y Fabiano et al. (2008) (9).

La aplicación del nuevo procedimiento, reducirá la demanda de este tipo de personal y por tanto disminuirán los índices de siniestralidad registrados por Miranda-García y Martínez-López (2007) (30). Pero por otra parte, cabría pensar, según los resultados obtenidos por Goffee 
(1996) (11), Stevens (1999) (12), Beaver (2003 (13), Fabiano et al. (2004) (14) y Halse et al. (2008) (15), que dicha reducción de personal podría llevar asociada una menor envergadura de las empresas de construcción de invernaderos, aumentando así la probabilidad de sufrir accidentes al ser más limitativa la aplicación de recursos preventivos en estas empresas. Estas dos afirmaciones, que en principio parecen contradictorias, en nuestro caso no lo son dado que los autores mencionados asocian siniestralidad con menor número de trabajadores, fundamentalmente, porque estas empresas de menor dimensión tienen más limitaciones en la incorporación de equipos y procedimientos preventivos. Sin embargo, esta asociación no es de aplicación en las empresas que apliquen el nuevo procedimiento constructivo propuesto, ya que aunque previsiblemente necesitarán menos trabajadores, ello no conllevará una limitación en los recursos preventivos, sino todo lo contrario, ya que se reduce la mano de obra precisamente por la incorporación de procedimientos constructivos más seguros.

La importante reducción en el consumo de plataformas elevadoras, 44,89\%, conlleva un importante ahorro económico, a la vez que se logra reducir la siniestralidad por atropello así como los riesgos derivados de su uso citados por Ponce-De León (2005) (31).

Además de reducir las horas de trabajo en altura, la mano de obra empleada y el uso de maquinaria complementaria, el nuevo procedimiento constructivo reduce en un 9,93\% el tiempo de montaje global del invernadero ensayado, debido a que los operarios tienen una mayor libertad de movimientos a nivel del sue- lo. Este resultado permite aumentar la disponibilidad de tiempo, evitando la ejecución de tareas forzadas con estrés, que como relata CallejónFerre, et al., (2009) (29) disminuyen la atención y aumentan la fatiga del trabajador, reduciendo de esta forma la siniestralidad asociada.

Finalmente, resulta destacable que los trabajadores tenían una gran experiencia constructiva con el método tradicional. Por ello, cabe esperar que cuando la adquieran con el nuevo procedimiento las ventajas serán aún más significativas.

\section{CONCLUSIONES}

Aunque los resultados del ensayo muestran una reducción de mano de obra, cifrada en un $14,74 \%$ en el caso de oficiales y un $7,65 \%$ en el caso de peones, una disminución del uso de plataformas elevadoras de un 44,89\%, una minoración del tiempo de montaje global del invernadero de un $9,93 \%$ y sus implicaciones en cuanto al ahorro de costes, la conclusión más importante de las obtenidas es la referente a la reducción de la siniestralidad por caídas en altura $(9,2 \%)$ motivada por la disminución de las horas de trabajo en altura en un $40 \%$.

\section{AGRADECIMIENTOS}

Los autores agradecen la financiación del presente trabajo de investigación mediante el Proyecto "Invermóvil" (código 2002000874), a la Consejería de Innovación, Ciencia y Empresa de la Junta de Andalucía, desarrollado en colaboración por Novedades Agrícolas S.A., Escuela Técnica de Investigación y Formación Agraria (ETIFA) y la Universidad de Almería.

\section{BIBLIOGRAFÍA}

(1) Von Elsner, B.; Briassoulis, D.; Waaijenberg, D.; Mistriotis, A.; Von Zabeltitz, C.; Gratraud, J.; Russo, G.; Suay-Cortes, R.: Review of structural and functional characteristics of greenhouses in European Union countries, part I: design requirements. Journal of Agricultural Engineering Research, Vol. 75, nº 1 (2000), pp.1-16. doi:10.1006/jaer.1999.0502.

(2) Castilla, N.; Hernández, J.: The plastic greenhouse industry of Spain. Chronica Horticulturae, Vol.45, no 3 (2000), pp. 15-20.

(3) Sanjuán, J. F.: Detección de la superficie invernada en la provincia de Almería a través de IMÁG. p.68, Ed. FIAPA, Almería, Spain, 2007.

(4) Camacho-Ferre, F.: Técnicas de producción de frutas y hortalizas en los cultivos protegidos del Sureste español, p. 250, Ed. Caja Rural de Almería, Almería, Spain, 1999.

(5) Soriano, T.; Montero, J. I.: Sanchez-Guerrero, M. C.; Medrano, E.; Anton, A.; Hernandez, J.: Morales, M. I.; Castilla, N.: A study of direct solar radiation transmission in asymmetrical multi-span greenhouses using scale models and simulation models. Biosystems Engineering, Vol. 88, $\mathrm{n}^{\circ} 2$ (2004), pp.243-253. doi:10.1016/j.biosystemseng.2004.03.006.

(6) López-Hernández, J. C.; Pérez-Parra, J.: Evolución de las estructuras de invernadero. Plasticulture, Vol. 125 (2006), pp. 8-17.

(7) Saha, A.; Ramnath, T.; Chaudhuri, R.; Saiyed, H.: An accident risk assessment study of temporary piece rated workers Indian. Indian Journal of Medical Sciences, Vol. 42, n 2 (2004), pp. 240-245.

(8) Waehrer, G.; Dong, X.; Miller, T.; Haile, E.; Men, Y.: Costs of occupational injuries in construc tion in the United States. Accident Analysis Prevention, Vol. 39, nº 6 (2004), pp. 1258-1266. doi:10.1016/j.aap.2007.03.012.

(9) Fabiano, B.; Curro, F.; Reverberi, A.; Pastorino, R.: A statistical study on temporary work and occupational accidents: Specific risk factors and risk management strategies. Safety Science, Vol. 46, $\mathrm{n}^{\circ} 3$ (2008), pp. 535-544. doi:10.1016/j.ssci.2007.05.004.

(10) Anuario de la agricultura almeriense. Agricultura 2000. Ed. Novotécnica, Almería, Spain, 2000.

(11) Goffee, R.: Understanding family businesses: issues for further research. International Journal of Entrepreneurial Behaviour and Research,Vol.2, nº1 (1996), pp. 36-48. doi:10.1108/13552559610110709. 
(12) Stevens, G.: Features-workplace injuries in small and large manufacturing workplaces -an analysis of the risks of fatal and non fatal injuries-, including figures for 1994/5-1995/6. Labour Market Trends, Vol. 107, no 1 (1999), pp. 19-26.

(13) Beaver, G.: Management and the small firm. Strategic Change, vol. 12 (2003), pp. 63-68.

(14) Fabiano, B.; Curro, F.; Pastorino, R.: A study of the relationship between occupational injuries and firm size and type in the Italian industry. Safety Science, Vol. 42, n 7 (2004), pp. 587-600. doi:10.1016/j.ssci.2003.09.003.

(15) Hasle, P.; Kines, P.; Andersen, L. P.: Small enterprise owner's accident causation attribution and prevention. Safety Science, Vol. 47, no 1 (2009), pp. 9-19. doi:10.1016/j.ssci.2007.12.005.

(16) Haslam, R. A.; Hide, S. A.; Gibb, A. G. F.; Gyi, D. E.; Pavitt, T.; Atkinson, S.; Duff, A. R.: Contributing factors in construction accidents. Applied Ergonomics, Vol. 36, no 4 (2005), pp. 401-415. doi:10.1016/j.apergo.2004.12.002.

(17) Vivian, W. Y. Tam.; Khoa, N. Le.: Quality improvement in construction by using a Vadermonde interpolation technique. International Journal of Project Management, Vol. 25, no 8 (2007), pp. 815-823. doi:10.1016/j.ijproman.2007.03.009.

(18) Zeng, S. X.; Vivian, W. Y. Tam.; Tam, C. M.: Towards occupational health and safety systems in the construction industry of China. Safety Science, Vol. 46, no 8 (2007), pp. 1155-1168. doi:10.1016/j. ssci.2007.08.005.

(19) Camino-López, M. A.; Ritzel, D. O.; Fontaneda, I.; González-Alcantara, O. J.: Construction industry accidents in Spain. Journal of Safety Research, Vol. 39, nº 5 (2008), pp. 497-507. doi:10.1016/j. jsr.2008.07.006.

(20) Lehtola, M.; Van Der Molen, H.; Lappalainen, J.; Hoonakker, P.; Hsiao, H.; Haslam, R.; Hale, A.; Verbeek, J.: The effectiveness of interventions for preventing injuries in the construction industry. A Systematic Review. American Journal of Preventive Medicine, Vol. 35, no 1 (2008), pp. 77-85. doi:10.1016/j.amepre.2008.03.030.

(21) Möller, N.; Hansson, S. O.: Principles of engineering safety: risk and uncertainty reduction. Reliability Engineering \& System Safety, Vol. 93 (2008), pp. 776-783. doi:10.1016/j.ress.2007.03.031.

(22) Pérez-Alonso, J.; Carreño-Ortega, A.; Callejón-Ferre, A. J.; Vázquez-Cabrera, F. J. : Preventive activity in the greenhouse-construction industry of south-eastern Spain. Safety Science, Vol. 49 (2011), pp. 345-354. doi:10.1016/jssci.2010.09.013.

(23) Pérez-Alonso, J.; Carreño-Ortega, A.; Vázquez-Cabrera, F. J.; Callejón-Ferre, A. J.: Accidents in the greenhouse-construction industry of SE Spain. Applied Ergonomics, Vol. 43 (2012), pp. 69-80. doi:10.1016/j.apergo.2011.03.007

(24) Hoxey, R.; Robertson, A.: Design developments in plastic greenhouses. Plasticulture, Vol. 97, nº 1 (1993), pp. 3-16.

(25) Matallana-González, A.; Oriol-Marfá, I.; Pagés, J.: Los invernaderos y la crisis energética. p. 132, Instituto Nacional de Investigaciones Agrarias, Madrid, Spain, 1980.

(26) Tsirogiannis, I. L.: Greenhouse types in Greece; causes of damage and failure. Diploma thesis, Dept. of Agricultural. Engineering, Agricultural University of Athens, Athens, Greece (in Greek), 1996.

(27) Waaijenberg, D.: Code of Practice for greenhouses with flexible claddings structural aspects. Report. 97-01, IMAGDLO, Wageningen, The Netherlands, 1997.

(28) Mistriotis, A.; Briassoulis, D.: Numerical estimation of the internal and external aerodynamic coefficients of a tunnel greenhouse structure with openings. Computers and Electronics in Agriculture, Vol. 34, no 1-3 (2002), pp. 191-205. doi:10.1016/S0168-1699(01)00187-9.

(29) Callejon-Ferre, A. J.; Perez-Alonso, J.; Sanchez-Hermosilla, J.; Carreño-Ortega, A.: Ergonomics and psycho-sociological quality indeces in greenhouses of Almería (Spain). Spanish Journal of Agricultural Research, Vol. 7, no 1 (2009), pp.50-58.

(30) Miranda-García, R.M., Martínez-López, D.: Estudio sobre los riesgos laborales derivados de la construcción de invernaderos y de las medidas preventivas aplicables para reducir su incidencia. p. 217, Colegio Oficial de Ingenieros Técnicos Agrícolas de Almería y Consejería de Empleo de la Junta dé Andalucía, Almería, Spain, 2007.

(31) Ponce-De León, J.L.: Comisión Nacional de Seguridad y Salud en el Trabajo. Grupo de trabajo Sector Agrario. Ministerio de Trabajo e Inmigración, Spain, 2005.

(32) Cristensen, W.: Safety through design. Professional Safety, Vol. March (2003), pp. 32-39.

(33) Behm, M.: Linking construction fatalities to the design for construction safety concept. Safety Science, Vol. 43, no 8 (2005), pp. 589-611. doi:10.1016/j.ssci.2005.04.002.

(34) Lam, P. T. I.; Wong, F. W. H.; Chan, A. P. C.: Contributions of designers to improving buildability and constructability. Design Studies, Vol. 27, nº 4 (2006), pp. 457-479. doi:10.1016/j.destud.2005.10.003.

(35) Mrosczyzk, J.: Designing for construction worker safety. ASSE Blueprints, Vol. 5, no 3 (2006), pp.1-4.

(36) Van Gorp, A.: Ethical issues in engineering design processes; regulative frameworks for safety and sustainability. Design Studies, Vol. 28, nº 2 (2007), pp. 117-131. doi:10.1016/j.destud.2006.11.002.

(37) Gambatese, J. A.; Behm, M.; Rajendran, S.: Design's role in construction accident causality and prevention: Perspectives from an expert panel. Safety Science, Vol. 46, no 4 (2007), pp. 675-691. doi:10.1016/j.ssci.2007.06.010.

(38) Toole, T. M.; Gambatese, J.: The trajectories of Construction Prevention through Design. Journal of Safety Research, Vol. 39, no 2 (2008), pp. 225-230. doi:10.1016/j.jsr.2008.02.026.

(39) Dzul, L. A.; Gracia, S.: Contexto actual de los sistemas de costes de la calidad desarrollados y aplicados a proyectos de construcción: la necesidad de medición de la calidad en el diseño. Informes de la Construcción, Vol. 61, no 514 (2009), pp. 41-50. doi: 10.3989/ic.07.004.

(40) Callejón-Ferre, A. J.; Camacho-Ferre, F.; López-Martínez, J. A, Simón-Domínguez, J. V.; GonzálezZapata, J.: Seguridad, higiene y salud en la construcción de invernaderos Tipo Almería. Horticultura, vol.130 (1998), pp. 66-69.

(41) Fernández, M.: Soporte para arcos de invernadero. № Publicación: 1037 894: Oficina Española de Patentes y Marcas. Modelo Utilidad. U 9701 988. 1998-01-05.

(42) Villegas, M.: Elementos Constructivos para invernaderos. No Publicación: 1043 347: Oficina española de patentes y marcas. Modelo Utilidad. U 009901 692.1999-28-06.

(43) Vázquez-Cabrera, F. J.; Pérez-Alonso, J.; Callejón-Ferre, A. J.; Carreño-Ortega, A.: Diseño de un nuevo capitel para invernaderos multitúnel. Informes de la Construcción, Vol. 63, 521 (2011), pp. 47-56. doi:10.3989/ic.09.009

(44) Gómez-Cano, M.: Evaluación de riesgos laborales (2 $2^{a}$ edición), p. 13, Instituto Nacional de Seguridad e Higiene en el Trabajo, Madrid, 1998. 